\title{
Can only flavor-nonsinglet $H$ dibaryons be stable against strong decays?
}

\author{
Stathes D. Paganis', Takeshi Udagawa', \\ G. W. Hoffmanne', and R. L. Ray由 \\ Department of Physics, University of Texas at Austin \\ Austin, Texas 78712 \\ Manuscript Prepared for Brief Reports of Physical Review C
}

\begin{abstract}
Using the QCD sum rule approach, we show that the flavor-nonsinglet $H$ dibaryon states with $\mathrm{J}^{\pi}=1^{+}, \mathrm{J}^{\pi}=0^{+}, \mathrm{I}=1$ (27plet) are nearly degenerate with the $\mathrm{J}^{\pi}=0^{+}, \mathrm{I}=0$ singlet $H_{0}$ dibaryon, which has been predicted to be stable against strong decay, but has not been observed. Our calculation, which does not require an instanton correction, suggests that the $H_{0}$ is slightly heavier than these flavor-nonsinglet $H$ s over a wide range of the parameter space. If the singlet $H_{0}$ mass lies above the $\Lambda \Lambda$ threshold $(2231 \mathrm{MeV})$, then the strong interaction breakup to $\Lambda \Lambda$ would produce a very broad resonance in the $\Lambda \Lambda$ invariant mass spectrum which would be very difficult to observe. On the other hand, if these flavor-nonsinglet $\mathrm{J}=0$ and $1 H$ dibaryons are also above the $\Lambda \Lambda$ threshold, but below the $\Xi^{0} n$ breakup threshold $(2254 \mathrm{MeV})$, then because the direct, strong interaction decay to the $\Lambda \Lambda$ channel is forbidden, these flavor-nonsinglet states might be more amenable to experimental observation. The present results allow a possible reconciliation between the reported observation of $\Lambda \Lambda$ hypernuclei, which argue against a stable $H_{0}$, and the possible existence of $H$ dibaryons in general.
\end{abstract}

PACS number(s): 12.40.Yx, 14.20.Pt

\footnotetext{
${ }^{1}$ e-mail address: paganis@physics.utexas.edu

${ }^{2}$ e-mail address: udagawa@physics.utexas.edu

${ }^{3}$ e-mail address: hoffmann@physics.utexas.edu

${ }^{4}$ e-mail address: ray@physics.utexas.edu
} 


\section{Introduction}

Using the MIT bag model, Jaffe[1] predicted a stable (against strong decay) sixquark flavor-singlet (uuddss) hadron (referred to as $H_{0}$ ) with $\mathrm{J}=0, \mathrm{I}=0$, and $\mathrm{S}=-2$. A plethora of mass calculations [2] followed Jaffe's work, and most of them predicted a weakly bound $H_{0}$ of mass just below the $\Lambda \Lambda$ threshold $(2231 \mathrm{MeV})$. For 20 years experiments have searched for the $H_{0}$, but no convincing evidence has been found for its existence [3, 4]. In fact, the candidate $H_{0}$ s from different experiments have very different masses. One such experiment [4] claims the observation of a very weakly bound $\Lambda \Lambda$ hypernucleus which excludes at some level the existence of the $H_{0}$.

SU(3) flavor-nonsinglet $H$ states are not usually discussed in the literature because of the expectation that they should be heavier than the $H_{0}$. This expectation is due to the assumption that the effective magnetic one-gluon-exchange between the valence quarks is most attractive for the flavor-singlet channel, making the $H_{0}$ the lightest state.

In this work we consider the masses of two nonsinglet, doubly strange six-quark states which occur in the baryon-octet $\otimes$ baryon-octet direct product space [5]. These include the $\mathrm{J}^{\pi}=1^{+}, \mathrm{I}=0 \mathrm{H}$ from the $\mathrm{J}=1 S U(3)_{f}$ octet, and the $\mathrm{I}=1, \mathrm{I}_{3}=0 \mathrm{H}$ from the $\mathrm{J}^{\pi}=0^{+}$27plet. Based on model-independent assumptions, we show that it is likely that the $H_{0}$ is nearly degenerate with these states, and the $H_{0}$ mass is slightly larger. This contradiction with the MIT bag model has been discussed elsewhere [6]. We study the mass ratios, rather than absolute masses, because the ratio carries smaller uncertainty and is more stable over a wide region of the parameter space. The QCD sum rule approach was used by Ioffe[7 to calculate the mass splitting in the baryonic decuplet.

The situation for these $H$ states is analogous to the case of the $\eta^{\prime}(I=0, J=0)$, $\rho(\mathrm{J}=1, \mathrm{I}=1)$ and $\omega(\mathrm{J}=1, \mathrm{I}=0)$, in which the $\eta^{\prime}$ is the heaviest. The extra mass is attributed to the $U(1)_{A}$ anomalous symmetry breaking of QCD, which is taken into account in model calculations using instantons. The method employed here does not need an instanton correction because as discussed in [8] the instanton effects (if any) are effectively included in the quark condensates.

If the singlet $H_{0}$ mass is greater than the $\Lambda \Lambda$ mass, then it should not have been observed in previous $H$-search experiments [3, 4]. If the nonsinglet $\mathrm{J}=1, \mathrm{I}=0$ (octet) $H$ mass is above the $\Lambda \Lambda$ threshold $(2231 \mathrm{MeV})$, but below the $\Xi^{0} n$ threshold $(2254 \mathrm{MeV})$, then it can only decay weakly or electromagnetically because the strong decay to $\Lambda \Lambda$ is not allowed. The situation is different for the $\mathrm{J}=0, \mathrm{I}=1$ (27plet) $H$ since it may isospin mix with the $H_{0}$ and the $\mathrm{J}=0, \mathrm{I}=0$ (27plet) $H$ to form physical states which strong decay to $\Lambda \Lambda$. However, for this case, if the mixing is small, experimental evidence for these $H$ s may be a narrow peak in the $\Lambda \Lambda$ invariant mass

spectrum. Such an observation would not contradict the observed $\Lambda \Lambda$ hypernucleus events [4. The candidate nonsinglet $\mathrm{J}=0, \mathrm{I}=1 \mathrm{H}$ s reported by Shabazian et al. [3] 
might be explained in this way.

In Sec. II we discuss the QCD sum rule method and then formulate sum rules for the $H_{0}$, the $\mathrm{J}=1, \mathrm{I}=0$ (octet) $H$ and the $\mathrm{J}=0, \mathrm{I}=1$ (27plet) $H$. In Sec. III we calculate the mass ratios $m_{H_{0}} / m_{H}$ over a wide range of the parameter space. Finally, in Sec. IV we discuss our results and some experimental issues and present our conclusions.

\section{II $\quad H$ Dibaryon Sum Rule}

In this section, the QCD sum rules [9] are formulated for the $H_{0}$, the $\mathrm{J}=1, \mathrm{I}=0$ (octet) $H$ and the $\mathrm{J}=0, \mathrm{I}=1, \mathrm{I}_{3}=0$ (27plet) $H$. We follow the method described in Ref. [6]. The $H_{0}$ current, $J_{H_{0}}(x)$, is a product of two baryonic currents, $J_{B}(x)$, which are written using the convention in [10] as

$$
J_{B}(x)=O_{1}^{q_{i} q_{j} q_{k}}(x)+t O_{2}^{q_{i} q_{j} q_{k}}(x),
$$

where,

$$
O_{1}^{q_{i} q_{j} q_{k}}(x)=\epsilon_{a b c} \cdot\left[\left(q_{i}^{T a}(x) C q_{j}^{b}(x)\right) \gamma_{5} q_{k}^{c}(x)\right]
$$

and

$$
O_{2}^{q_{i} q_{j} q_{k}}(x)=\epsilon_{a b c} \cdot\left[\left(q_{i}^{T a}(x) C \gamma_{5} q_{j}^{b}(x)\right) q_{k}^{c}(x)\right] .
$$

In Eqs. (2) and (3) $q$ is the quark field, $i, j, k$ are flavor indices, $a, b, c$ are color indices, $C$ is the charge conjugation matrix, and the parameter $t$ is a weighting

coefficient for the second term of the baryonic current which is commonly used in QCD sum rule calculations. The $H_{0}$ dibaryon singlet current is given by

$$
J_{H_{0}}(x)=\left(2 \epsilon_{i j k^{\prime}} \epsilon_{i^{\prime} j^{\prime} k}-\frac{2}{3} \epsilon_{i j k} \epsilon_{i^{\prime} j^{\prime} k^{\prime}}\right) \cdot J_{B} C \gamma_{5} J_{B}
$$

where the antisymmetrization tensors, $\epsilon_{i j k}$, produce a color and flavor-singlet 6 -quark (uuddss) state with the quantum numbers of the $H_{0}$.

The current correlation function is written as

$$
\Pi_{H}\left(q^{2}\right) \equiv-i \int d^{4} x e^{i q x}\left\langle 0\left|T J_{H}(x) \bar{J}_{H}(0)\right| 0\right\rangle .
$$

We apply the operator product expansion (OPE) to Eq. (5) to obtain the right hand side (rhs) of the $H$ dibaryon sum rule for large $Q^{2}=-q^{2}$. The advantage of the $\mathrm{OPE}$ for dibaryons is that there are just three non-vanishing terms that give large contributions in the $S U(3)_{f}$ limit. The resulting correlation function in the $S U(3)_{f}$ limit is 6

$$
\begin{aligned}
\Pi_{H}\left(q^{2}\right)= & \frac{h_{1}(t)}{2^{14} \pi^{10} \Gamma(9) \Gamma(8)}\left(-q^{2}\right)^{7} \ln \left(-q^{2}\right)+\frac{h_{2}(t)}{2^{8} \pi^{6} \Gamma(6) \Gamma(5)}\left(-q^{2}\right)^{4} \ln \left(-q^{2}\right) \cdot \frac{\langle\bar{q} q\rangle^{2}}{\left(4 N_{c}\right)^{2}} \\
& +\frac{h_{3}(t)}{2^{2} \pi^{2} \Gamma(3) \Gamma(2)}\left(-q^{2}\right) \ln \left(-q^{2}\right) \cdot \frac{\langle\bar{q} q\rangle^{4}}{\left(4 N_{c}\right)^{4}}
\end{aligned}
$$


where $N_{c}$ is the number of color charges and $\langle\bar{q} q\rangle$ is the quark condensate. The $h_{i}(t)$ coefficients in Eq. (6) are obtained by calculating all the possible contractions in Eq. (5). For example, for the $h_{1}(t)$ and $t=0$, there are 3600 non-vanishing terms. The $h_{i}(t)$ s for the $H_{0}$ have been calculated elsewhere [6].

Using the group symmetry properties of the dibaryon octet $\otimes$ octet direct product space [5] the current for the $\mathrm{J}=0, \mathrm{I}=1, \mathrm{I}_{3}=0$ (27plet) $H$ is written as

$$
\begin{aligned}
J_{H^{27}, I=1, I_{3}=0}(x)= & 2 J_{\Lambda} C \gamma^{5} J_{\Sigma^{0}}+2 J_{\Sigma^{0}} C \gamma^{5} J_{\Lambda} \\
& +J_{P} C \gamma^{5} J_{\Xi^{-}}+J_{\Xi^{-}} C \gamma^{5} J_{P} \\
& -J_{N} C \gamma^{5} J_{\Xi^{0}}-J_{\Xi^{0}} C \gamma^{5} J_{N},
\end{aligned}
$$

where for the baryon currents we use the convention of Eq. (11). For example, $P=\left(q_{i} q_{j} q_{k}\right)=(u d u)$ for the proton. The calculation yields the following $h_{i}$ coefficients:

$$
\begin{aligned}
& h_{1}(t)=1302+120 t-804 t^{2}+984 t^{3}+858 t^{4} \\
& h_{2}(t)=5208+480 t-3216 t^{2}+3936 t^{3}-6408 t^{4} \\
& h_{3}(t)=-20832-1920 t+12864 t^{2}-15744 t^{3}+25632 t^{4} .
\end{aligned}
$$

For the $\mathrm{J}^{\pi}=1^{+}, \mathrm{I}=0$ (octet) $H$ the current is written as

$$
\begin{aligned}
J_{H^{*}}^{\mu}(x)= & J_{\Sigma^{0}} C \gamma^{\mu} J_{\Sigma^{0}}-\frac{1}{2} J_{\Sigma^{+}} C \gamma^{\mu} J_{\Sigma^{-}}-\frac{1}{2} J_{\Sigma^{-}} C \gamma^{\mu} J_{\Sigma^{+}} \\
& -\frac{1}{3} J_{\Lambda} C \gamma^{\mu} J_{\Lambda}+\frac{1}{4} J_{N} C \gamma^{\mu} J_{\Xi^{0}}+\frac{1}{4} J_{\Xi^{0}} C \gamma^{\mu} J_{N} \\
& +\frac{1}{4} J_{P} C \gamma^{\mu} J_{\Xi^{-}}+\frac{1}{4} J_{\Xi^{-}} C \gamma^{\mu} J_{P} .
\end{aligned}
$$

This state, called the $H^{*}$, does not strongly couple to the $\Lambda \Lambda$ channel [11, 12]. For this case the $h_{i}$ coefficients were determined to be:

$$
\begin{aligned}
& h_{1}(t)=1866+2087 t-365 t^{2}+1418 t^{3}+2636 t^{4} \\
& h_{2}(t)=7466+8349 t-1460 t^{2}+5671 t^{3}-20026 t^{4} \\
& h_{3}(t)=-29864-33397 t+5840 t^{2}-22683 t^{3}+80104 t^{4} .
\end{aligned}
$$




\section{Calculation of the Mass Ratios}

The $H_{0}$ mass is given[6] by the expression

$$
\begin{aligned}
m_{H}^{2}\left(M^{2}\right)= & {\left[\frac{h_{1}(t)}{2^{14} \pi^{10} \Gamma(8)}\left(M^{2}\right)^{9}\left(1-\Sigma_{8}\right)-\frac{h_{2}(t)}{2^{8} \pi^{6} \Gamma(5)} \cdot \frac{\langle\bar{q} q\rangle^{2}}{\left(4 N_{c}\right)^{2}}\left(M^{2}\right)^{6}\left(1-\Sigma_{5}\right)\right.} \\
& \left.+\frac{h_{3}(t)}{2^{2} \pi^{2} \Gamma(2)} \cdot \frac{\langle\bar{q} q\rangle^{4}}{\left(4 N_{c}\right)^{4}}\left(M^{2}\right)^{3}\left(1-\Sigma_{2}\right)\right] / \\
& {\left[\frac{h_{1}(t)}{2^{14} \pi^{10} \Gamma(9)}\left(M^{2}\right)^{8}\left(1-\Sigma_{7}\right)-\frac{h_{2}(t)}{2^{8} \pi^{6} \Gamma(6)} \cdot \frac{\langle\bar{q} q\rangle^{2}}{\left(4 N_{c}\right)^{2}}\left(M^{2}\right)^{5}\left(1-\Sigma_{4}\right)\right.} \\
& \left.+\frac{h_{3}(t)}{2^{2} \pi^{2} \Gamma(3)} \cdot \frac{\langle\bar{q} q\rangle^{4}}{\left(4 N_{c}\right)^{4}}\left(M^{2}\right)^{2}\left(1-\Sigma_{1}\right)\right]
\end{aligned}
$$

where

$$
\Sigma_{i}=\sum_{k=0}^{i} \frac{s_{0}^{k}}{\left(M^{2}\right)^{k} k !} e^{-s_{0} / M^{2}},
$$

accounts for the continuum part, $M$ is the Borel mass, and $s_{0}$ is the continuum threshold. [ Eq. (11) also holds for the other $H \mathrm{~s}$, so the mass ratios, given by

$$
R=\frac{m_{H_{0}}\left(t, M_{0}^{(1)}, s_{0}^{(1)},\langle\bar{q} q\rangle^{2}\right)}{m_{H, J}\left(t, M_{0}^{(2)}, s_{0}^{(2)},\langle\bar{q} q\rangle^{2}\right)},
$$

were calculated for $\mathrm{J}=0$ and $\mathrm{J}=1$.

We use the standard assumptions for the phenomenological side (lhs) of Eq. (5) (pole term plus continuum for the spectral density, with continuum threshold $s_{0}$ ). The central values for our parameters are $t=-1.2, s_{0}=5.694 \mathrm{GeV}^{2}, M=1.5 \mathrm{GeV}$, $\langle\bar{q} q\rangle^{2}=(-0.250)^{3} \mathrm{GeV}^{3}$. We expect the calculation to be reliable for a wide range of the Borel mass $\mathrm{M}$ because the $H$ mass is determined by the chiral symmetry breaking scale $(\sim 1 \mathrm{GeV})$ 13 which is much larger than the scale at which QCD vacuum fluctuations become large $\left(\Lambda_{Q C D} \simeq 200 \mathrm{MeV}\right)$ and where the Borel smearing fails as it does for the case of the light pseudoscalar mesons. This region is taken to be around $2 \mathrm{GeV}$, where the higher order terms in the OPE are strongly suppressed and the pole dominates the continuum contribution. Our choice for the parameter $t$, as discussed in [8], gives self-consistent QCD sum rules which do not need an instanton correction. Instead, the instanton effects are adequately accounted for in the nonperturbative vacuum condensates. The Borel mass $M$ is in general different for the two $H$ states, but we expect it to be the same for degenerate states with the same quark content.

\footnotetext{
${ }^{5}$ The third power in the $M^{2}$ term in Eq. (11) is missing in Eqs. (10) and (15) of Ref. [6] due to a typographical error.
} 


\section{Results and Discussion}

Our results for the mass ratios from Eq. (12) and their sensitivities to variations of the input parameters are summarized in Fig. 1. Fig. 17a shows the dependence of the mass ratios on the Borel mass. The solid curve is $m_{H_{0}} / m_{H, J=0}$ (27plet), while the dashed curve is $m_{H_{0}} / m_{H, J=1}$ (octet). The ratios remain constant, and are slightly greater than 1, for a large range of the Borel mass. Fig. Ib shows the dependence of the mass ratios on the quark condensate. The curves have the same meaning as in Fig. 1a. Fig. 1]b shows that large variation of the quark condensate, within a range consistent with that found in the literature, produces very little effect $(\leq 2$ $\mathrm{MeV}$ for the mass difference). Fig. 1 $\mathrm{c}$ and Fig. 1 $\mathrm{d}$ show the mass ratio dependence on the continuum threshold $s_{0}$ and the parameter $t$. Again we see little sensitivity, about $0.1 \%$. Finally, the sensitivity of the mass ratio with respect to different Borel masses for the $H_{0}$ and the $\mathrm{J}=0, \mathrm{I}=1$ (27plet) $H$ or the $\mathrm{J}=1, \mathrm{I}=0$ (octet) $H$ was studied. The Borel mass of the $H_{0}$ was fixed and the nonsinglet $H$ Borel mass was varied. These mass ratios were essentially the same at each Borel mass and they decreased linearly from 1.05 for a Borel mass of $1.3 \mathrm{GeV}$ to 0.99 for a Borel mass of $1.6 \mathrm{GeV}$. Other contributors to the theoretical uncertainty are the neglected terms in the OPE (which we expect to be small) and the vacuum saturation assumption: $\left\langle\bar{q}^{2} q^{2}\right\rangle \sim\langle\bar{q} q\rangle^{2},\left\langle\bar{q}^{4} q^{4}\right\rangle \sim\langle\bar{q} q\rangle^{4}$

Our conclusion, based on the results presented in Fig. 1 is that the mass ratio remains very close to unity in the parameter range where we believe the calculation to be reliable. Both the $\mathrm{J}=1, \mathrm{I}=0$ (octet) $H$ and the $\mathrm{J}=0, \mathrm{I}=1$ (27plet) $H$ are almost degenerate with the $H_{0}$, and this degeneracy is not sensitive to the various QCD sum rule parameters. The mass difference is of the order of $0.1 \%$ for both $H$ states, or about $2 \mathrm{MeV}$ for $m_{H}=2 \mathrm{GeV}$. Surprisingly, we find that the singlet $H_{0}$ is slightly heavier than both the $\mathrm{J}=0, \mathrm{I}=1$ (27plet) $H$ and the $\mathrm{J}=1, \mathrm{I}=0$ (octet) $H$.

It is worthwhile to consider the consequences of these predictions for experiments designed to search for neutral, strange dibaryons. We note that the nonsinglet $\mathrm{J}^{\pi}=1^{+}, \mathrm{I}=0$ octet $H$ cannot strong decay to $\Lambda \Lambda$ (due to angular momentum and parity conservation), and if its mass is lower than the $\Xi^{0} \mathrm{n}$ threshold $(2254 \mathrm{MeV})$, then it may only decay electromagnetically (via an M1 transition to the $\mathrm{J}^{\pi}=0^{+}$, I=1 27plet $H$ and/or the singlet $H_{0}$ if these are lower in mass, or to the $\Lambda \Lambda \gamma$ channel) or weakly (if its mass is below that of the other $H \mathrm{~s}$ and the $\Lambda \Lambda$ threshold). If the $\mathrm{J}^{\pi}=0^{+}, \mathrm{I}=127$ plet $H$ has mass greater than $2231 \mathrm{MeV}$ it cannot strong decay to $\Lambda \Lambda$ due to isospin conservation. However, isospin mixing, due to electromagnetic interactions within the dibaryon, cause the physical $\mathrm{J}^{\pi}=0^{+} 27$ plet $H$ to contain a small $\mathrm{I}=0$ admixture, which allows strong decay to $\Lambda \Lambda$. If the admixture is small, then the state could have a narrow width of perhaps a few $\mathrm{MeV}$. The 27 plet $H$ cannot electromagnetically decay directly to the singlet $H_{0}$ (except via two-photon decay), however an E1 transition to the $\Lambda \Lambda \gamma$ is permitted. If the $\mathrm{J}=0$ 27plet $H$ is below the other $H$ s and the $\Lambda \Lambda$ threshold, then it may only decay by the weak 
interaction.

If we assume the predicted mass ratios found here and that each mass is between the $\Lambda \Lambda$ mass and the $\Xi^{0} \mathrm{n}$ mass, then the strong and electromagnetic decay schemes for the three $H \mathrm{~s}$ will be as shown in Fig. 2. If the decay of the $\mathrm{J}^{\pi}=0^{+} 27$ plet $H$ is driven by the strong decay channel via the small isospin mixing $\mathrm{I}=0$ component rather than by the electromagnetic decay to the $\Lambda \Lambda \gamma$ channel, then a relatively narrow peak should appear in the $\Lambda \Lambda$ invariant mass spectrum between 2231 and $2254 \mathrm{MeV}$. The decay of the $\mathrm{J}^{\pi}=1^{+}, \mathrm{I}=0$ octet $H$ to the $\Lambda \Lambda \gamma$ channel would result in a broad three-body phase space distribution for the $\Lambda \Lambda$ mass spectrum if the photon is not observed. The electromagnetic decays are as follows: (1) $\mathrm{J}^{\pi}=1^{+}$, $\mathrm{I}=0$ octet $H$ to the $\mathrm{J}^{\pi}=0^{+} 27$ plet $H$ is a $\Delta \mathrm{I}=0$ and $1, \mathrm{M} 1$ transition, (2) $\mathrm{J}^{\pi}=1^{+}$, $\mathrm{I}=0$ octet $H$ to $\Lambda \Lambda \gamma$ occurs via $\mathrm{E} 1$ and $\mathrm{M} 1$ transitions (all are $\Delta \mathrm{I}=0$ ), and (3) $\mathrm{J}^{\pi}=0^{+} 27$ plet $H$ to $\Lambda \Lambda \gamma$ occurs via an E1 transition. In Fig. 2 the strong decay of the $H_{0}$ is indicated by the thick, solid arrow, the strong decay via the small isospin mixing component is shown by the thick, dashed arrow, and the electromagnetic decays by the thin, solid arrows.

Observation of these nonsinglet $H$ s would not contradict the $\Lambda \Lambda$ hypernucleus events already observed [4]. It is possible that the candidate nonsinglet $H(\mathrm{~J}=0$, $\mathrm{I}=1$ ) observed by Shabazian et al. [3] can be explained by these results since we predict the $\mathrm{J}^{\pi}=0^{+} 27$ plet $H$ to be approximately degenerate with $H_{0}$. However, the mass must be less than that reported in [3] in order to be below the $\Xi^{0} \mathrm{n}$ breakup threshold. Clearly the discovery of flavor-nonsinglet $H$ dibaryons would require us to revisit traditional hadronic structure models and require a better understanding of quark-quark effective interactions.

The authors thank Dr. Ron Longacre at the Brookhaven National Laboratory for helpful discussions. This work was supported in part by the U.S. Department of Energy and The Robert A. Welch Foundation. 


\section{References}

[1] R. L. Jaffe, Phys. Rev. Lett. 38 (1977) 195.

[2] R. L. Jaffe and C. L. Korpa, Nucl. Phys. B258 (1985) 468; Y. Iwasaki, T. Yoshie and Y. Tsuboi, Phys. Rev. Lett. 60 (1988) 1371; M. Oka, K. Shimizu and K. Yazaki, Phys. Lett. B130 (1983) 365; Nucl. Phys. A464 (1987) 700; M. Oka, Phys. Rev. D38 (1988) 298; A. Faessler and U. Straub, Phys. Lett. B183 (1987) 10; U. Straub, Z. Zhang, K. Brauer, A. Faessler, and S. B. Khudkikar, Phys. Lett. B200 (1988) 241; Y. Koike, K. Shimizu and K. Yazaki, Nucl. Phys. A513 (1990) 653.

[3] J. Belz et al., Phys. Rev. Lett. 76 (1996) 3277; J.K. Ahn et al., Phys. Lett. B378 (1996) 53; B. A. Shabazian, T. A. Volokhovskaya, V. N. Emelyanenkjo, and A. S. Martynov, Phys. Lett. B316 (1993) 593; K. Imai, Nucl. Phys. A527 (1991) 181c.

[4] S. Aoki et al., Prog. Theor. Phys. 85 (1991) 1287.

[5] S.-Q. Xie, J. Phys. G: Nucl. Part. Phys. 15 (1989) 287.

[6] N. Kodama, M. Oka and T. Hatsuda, Nucl. Phys. A580 (1994) 445.

[7] B. L. Ioffe, Nucl. Phys. B188 (1981) 317; ibid. B191 (1981) 591.

[8] D. B. Leinweber, SLAC-SPIRES nucl-th/9510051 (1996); accepted for publication in Annals of Phys.

[9] M. A. Shifman, A. I. Vainshtein and V. I. Zakharov, Nucl. Phys. B147 (1979) 385; ibid. B147 (1979) 448.

[10] D. Espriu, P. Pascual and R. Tarrach, Nucl. Phys. B214 (1983) 285.

[11] R. P. Bickerstaff and B. G. Wybourne, J. Phys. G: Nucl. Phys. 7 (1981) 275.

[12] R. L. Jaffe, Phys. Rev. Lett. 38 (1977) 617.

[13] Aneesh Manohar and Howard Georgi, Nucl. Phys. B234 (1984) 189. 


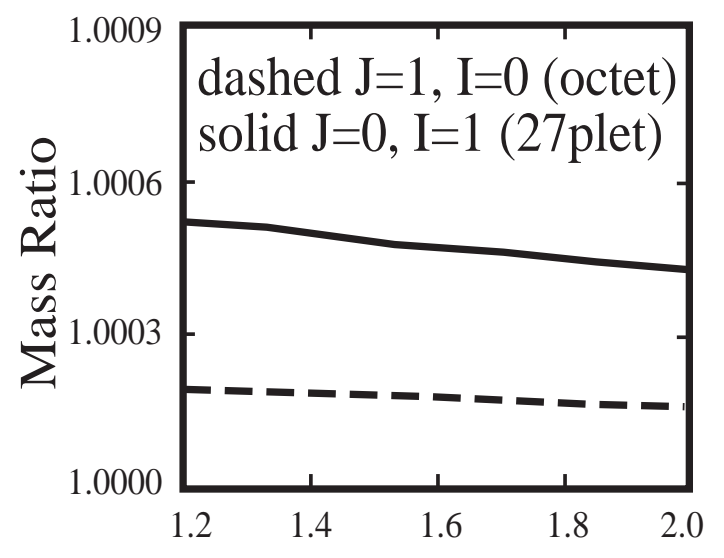

(a) Borel Mass $(\mathrm{GeV})$

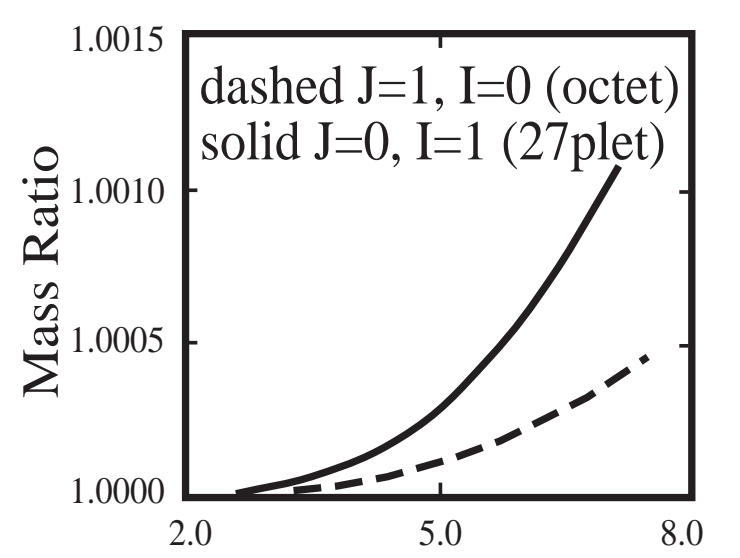

(c) Vacuum Threshold $\mathrm{s}_{\mathrm{o}}(\mathrm{GeV})$
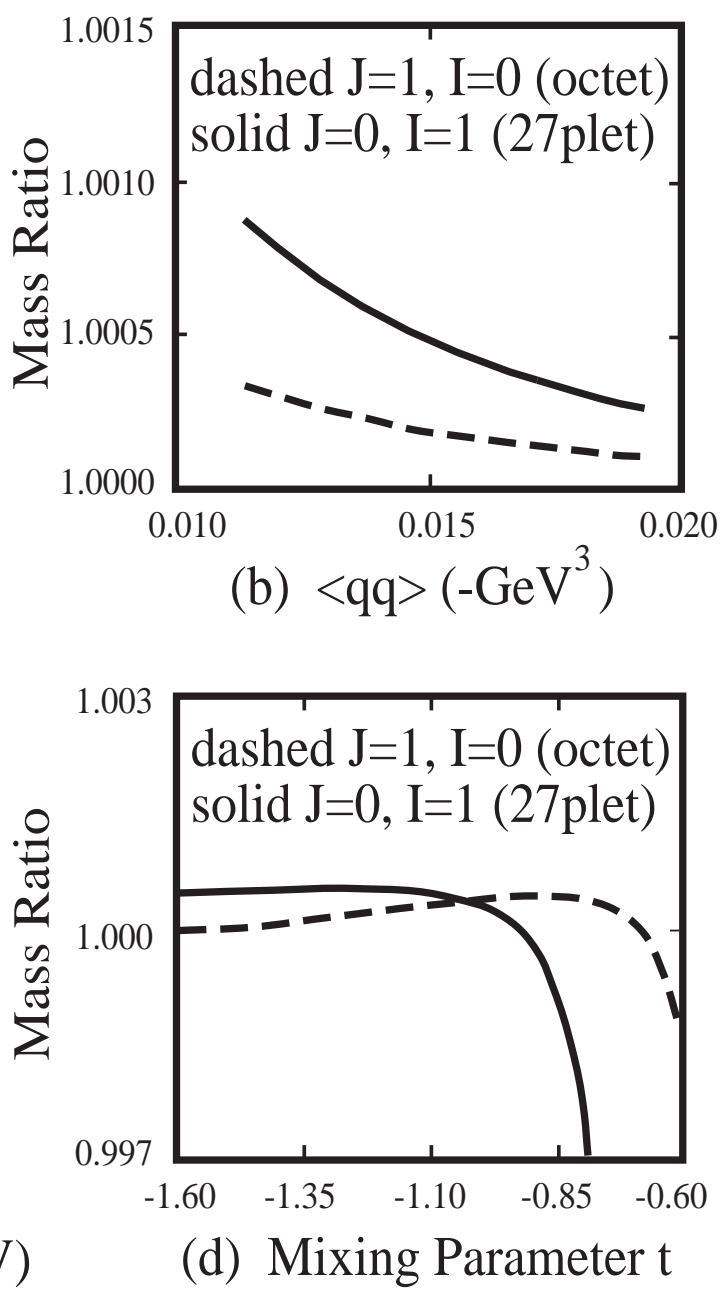

Figure 1: Dependence of the mass ratio [Eq. (12)] on (a) the Borel mass, (b) the quark condensate, (c) the vacuum threshold, and (d) the mixing parameter. The solid curve is $m_{H_{0}} / m_{H, J=0}$ (27plet), while the dashed curve is $m_{H_{0}} / m_{H, J=1}$ (octet). 


\section{H Dibaryon Decay Schemes}

$\Xi \mathrm{N}(2254 \mathrm{MeV})$

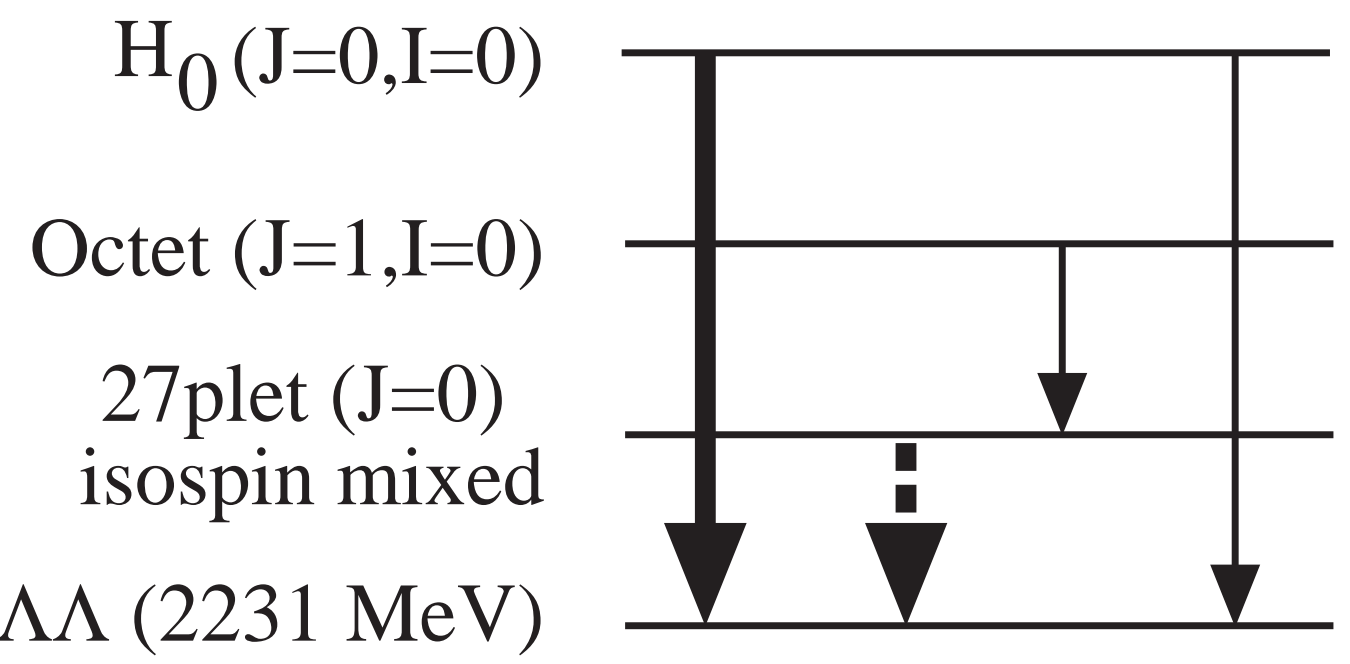

Figure 2: Strong and electromagnetic decay schemes for the $H_{0}$, the $\mathrm{J}=1, \mathrm{I}=0$ octet $H$, and the $\mathrm{J}=0$ isospin mixed 27plet $H$ assuming the relative masses predicted here and assuming the $H$ masses lie between 2231 and $2254 \mathrm{MeV}$, as discussed in the text. Thick, solid arrow indicates strong decay; thick, dashed arrow indicates strong decay via small $\mathrm{I}=0$ isospin admixture; and thin arrows indicate electromagnetic decays. 